\title{
Experimental performance analysis of a multiple-source and multiple-use heat pump system: a predictive ANN model of sky- source heat pump
}

\author{
Ke $\mathrm{WEN}^{1 *}$, Ryozo OOKA ${ }^{2}$, Toshiyuki $\mathrm{HINO}^{2}$, Mingzhe $\mathrm{LIU}^{1}$, Doyun $\mathrm{LEE}^{1}$, Wonjun $\mathrm{CHOI}^{2}$, Shintaro $\mathrm{IKEDA}^{3}$, and Djafar Reza \\ PALASZ $^{4}$ \\ ${ }^{1}$ Graduate School of Engineering, The University of Tokyo, 4-6-1 Komaba, Meguro-ku, Tokyo 153-8505, Japan \\ ${ }^{2}$ Institute of Industrial Science, The University of Tokyo, 4-6-1 Komaba, Meguro-ku, Tokyo 153-8505, Japan \\ ${ }^{3}$ Tokyo University of Science, 6-3-1, Niijuku, Katsushika-ku, Tokyo 125-8585, Japan \\ ${ }^{4}$ Technical University of Denmark, Anker Engelunds Vej 1 Bygning 101A, 2800 Kgs. Lyngby, Denmark
}

\begin{abstract}
In this study, an artificial neural network (ANN) was used to model the thermal performance of a novel direct-expansion solar-assisted sky-source heat pump (SSHP) during winter. The input parameters of the ANN take into account the weather conditions, water loop characteristics, and the compressor characteristics of the SSHP. The following four output parameters were adopted to evaluate the SSHP performance: the outlet water temperature of the water loop, electricity consumption, heat production, and the coefficient of performance. To increase the accuracy of the ANN and simultaneously investigate the effects of each of the input parameters on the performance of the SSHP, the combination of input parameters for the validation data set was varied in multiple case studies. Additionally, learning curves were introduced to clarify the relationship between the training data size and the generalization performance of the ANN. Finally, the ANNs with the best performance were selected and evaluated based on the test data set by using metrics such as the root mean square error. The reported results demonstrated that the ANN model has comparatively high SSHP winter performance prediction accuracy.
\end{abstract}

\section{Introduction}

Renewable energy has recently been receiving an increasing amount of attention because of increasing energy needs and the need to reduce greenhouse gas emissions [1]. There are multiple types of renewable energies that can be exploited as thermal resources for buildings, such as solar radiation and ground heat. However, every type of energy has its own advantages and disadvantages. For example, solar radiation is an abundant energy flow, but it has intermittent availability [2]. Hence, how to efficiently employ multiple types of renewable energies is becoming a difficult challenge. One of the usual solutions is to build a hybrid system integrating the supplementary components (e.g. solar-assisted heat pump) with a ground source heat pump [3-4]. Dai et al. carried out an experimental analysis of a solar assisted ground source heat pump system (SAGSHPS). Their results showed that the introduction of solar heat has a positive effect on the soil temperature recovery rate compared with the traditional ground source heat pump system [4]. However, the previous research mostly employ two renewable energy (e.g. solar energy and geothermal energy). Moreover, those hybrid system are usually designed for a single purpose (e.g. domestic hot water). Based on well-known information, we developed a multiple-source and multiple-use heat pump system (MMHP) system. This is a distributed water-source heat pump system that can utilize various types of renewable energies surrounding a building to meet a variety of thermal demands [2]. The system consists of a water loop that exchanges heat with a sky-source heat pump (SSHP), watersource heat pumps for various heating and cooling purposes, and a ground heat exchanger (GHE) [2]. To verify its practicability and effects, a small experimental building (hereafter referred to as RE house) was constructed in 2017. In a previous report, this heat supply system and a detailed experimental analysis of the thermal performance of a skysource heat pump (SSHP) were described [5]. Although the SSHP is one kind of direct-expansion solar-assisted heat pump (DXSAHP), compared with traditional DXSAHP, it has extruded aluminium finned which is exposed to ambient environment on the opposite face of the outdoor panel instead of being covered with insulation. On the one hand, this unique design help the SSHP not only absorb heat from solar radiation, but also additional heat from the ambient air through natural convection and wind in winter. On the other hand, it can also help the same panel enhance dissipating heat when the panel works as a nocturnal radiator through nocturnal radiation and natural draft in the summer night [6]. Thus, the SSHP is one of the most important components of this heat source system and being able to predict the

* Corresponding author: wkyai@iis.u-tokyo.ac.jp 
performance of the SSHP is critical to improving not only the SSHP operation efficiency but also the overall system operation efficiency. Generally, models used to predict the performance of HVAC equipment can be classified as either a black-box model or physics-based model. Unlike the physics-based model, the black-box model can easily establish relationships between the inputs and outputs with good prediction accuracy in the absence of any domain knowledge. Moreover, the black-box model has a low computational cost, and its weights can be adjusted for compatibility with the dynamic characteristics of HVAC equipment based on the collected data [7]. Artificial neural network (ANN) is one of the most widely used black-box models because of its ability to approximate complicated nonlinear relationships when a large amount of data is available. Some researchers have used ANN to predict the thermal performance of traditional DXSAHP. Mohanraj et al. used an ANN to predict the performance of a DXSAHP in winter [8]. Because their experiment was conducted under steady-state conditions, the input parameters of the ANN were generalized and categorized as either solar radiation or outdoor temperature parameters, and no other factors were considered. Moreover, the data sets used to train and test the ANN were limited to 50 and 10, respectively, which can be viewed as insufficient, as a small data set usually results in poor generalization of the trained ANN especially when the number of data is less than the number of weights and biases in ANN used. Gunasekar et al. developed an ANN to predict the energy performance of a photovoltaic-thermal evaporator used in solar assisted heat pumps and applied analysis of variance to identify the significant ambient parameter influencing the energy performance. The results showed that solar intensity and ambient temperature are the most influencing parameters while ambient wind velocity and ambient relative humidity had a less effect on the energy performance [9]. Having gone through above literature, it is observed that no related reports on using ANN to predict the thermal performance of a novel direct-expansion solarassisted heat pump such as SSHP. Therefore, in this study, an ANN was constructed based on the large amount of data collected from the previous winter field experiment, and subsequently used to model the performance of an SSHP. Additionally, to ensure that the ANN achieves high accuracy, and to investigate the effects of different input parameters on the performance of the SSHP, case studies were performed for different combinations of the input parameters. To begin, in Section 2, we will explain the full system, with particular focus on the SSHP, and the experiment. Then, in Section 3, we will provide a basic description of the ANN, the input and output parameters to be implemented in the ANN, and the development of the ANN model. Section 4 focuses on the results and discussion, and Section 5 summarizes this study.

\section{Experiments}

\subsection{Description of a multiple-source and multiple- use heat pump system}

The direct-expansion solar assisted SSHP is one of the most important components of a multiple-source and multiple-use heat pump (MMHP) system, which consists of a ground heat exchanger (GHE) and various water-source heat pumps besides the SSHP. All components are interconnected via a water-circulating loop to form a network that can transfer thermal energy. Figure 1 depicts this heat supply system. It is expected to systematically and efficiently exploit multiple types of natural renewable energies that can be derived from terrestrial and solar radiation sources to diversify thermal utilization. To verify its practicability and effects on a small experimental building, the RE house was constructed at the Kashiwa Campus of the University of Tokyo in 2017, as shown in Figure 2. For an in-depth introduction to the MMHP system and the RE house, refer to [2, 4].

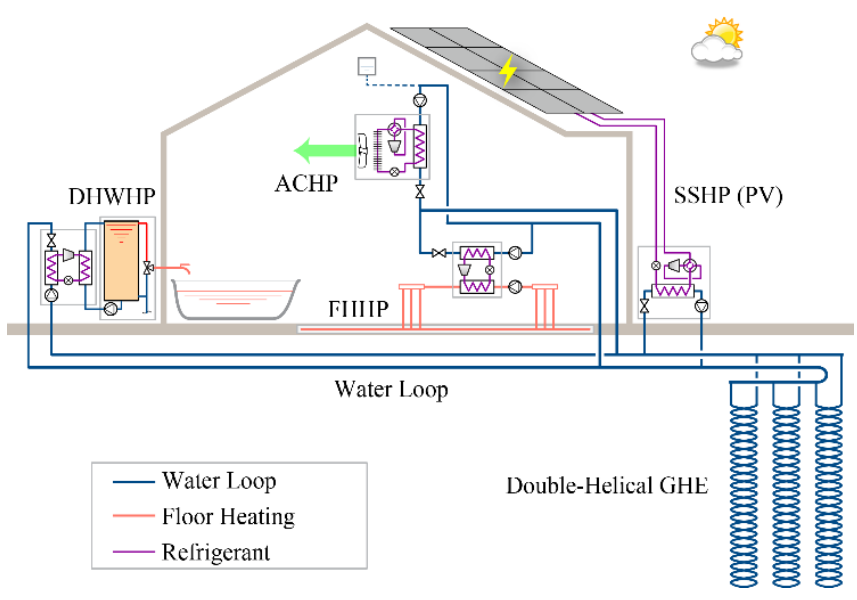

Fig. 1. Heat-supply system diagram of the RE house

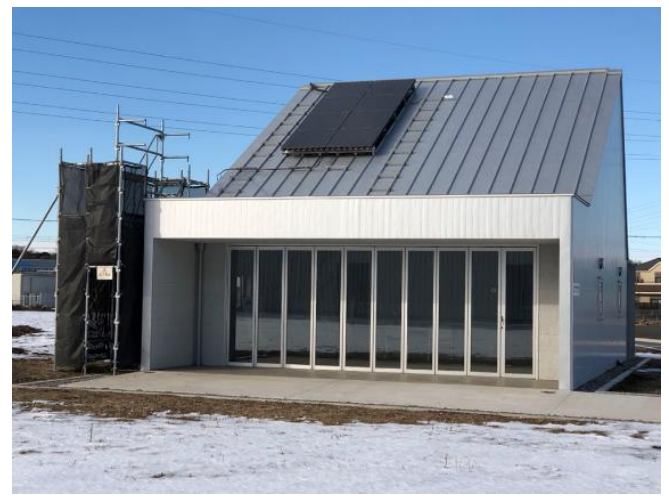

Fig. 2. RE house

\subsection{Description of the SSHP}

Figure 3 shows the refrigerant circuit of the SSHP module. It mainly consists of a compressor unit, an outdoor solar collector panel, and a plate-type heat exchanger. All of these main components are connected through a refrigerant circuit via R32. In winter, the heat exchanger works as a condenser for the SSHP by condensing the gaseous refrigerant flowing through the exchanger to dissipate heat into the water loop. The SSHP panel is formed by several finned tubes placed in parallel. Figure 4 shows the cross section of the SSHP outdoor panel. The top of the panel is an unglazed surface on which photovoltaic cells are attached to generate electricity; 
the opposite face of the panel has extruded aluminum finned tubes to further improve the heat transfer between the outdoor environment and the refrigerant passage. In winter, the SSHP panel works as an evaporator by evaporating the liquid refrigerant flowing through the refrigerant passage in order to collect heat from ambient air and solar radiation. When solar radiation is not sufficiently available to meet the required thermal output, the evaporation temperature falls lower than that of the ambient air in order to extract additional heat from the ambient air. When solar radiation increases, the refrigerant preferentially absorbs heat from solar radiation to make the surface temperature of the SSHP panel near or higher than that of the ambient air. The specifications of the SSHP module are presented in Table 1. For an in-depth introduction to the SSHP system, refer to $[2,4]$.

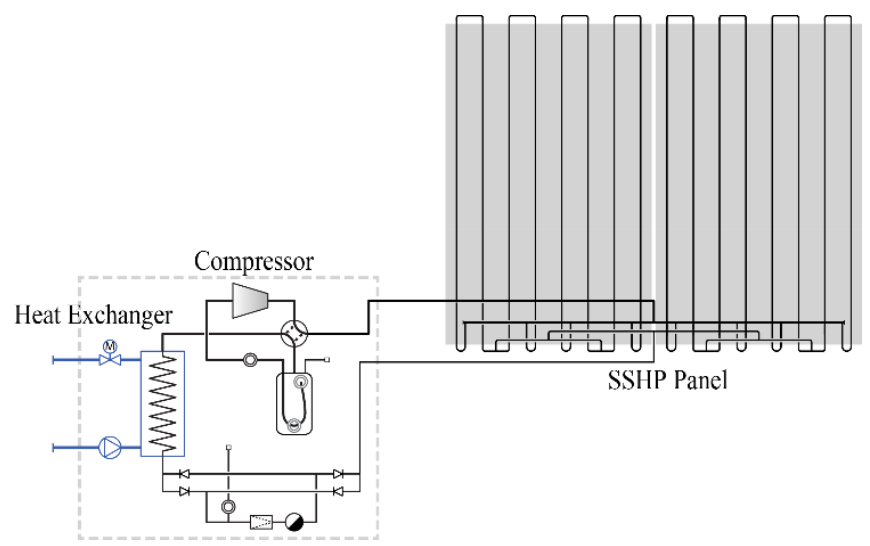

Fig. 3. Refrigerant circuit of SSHP module

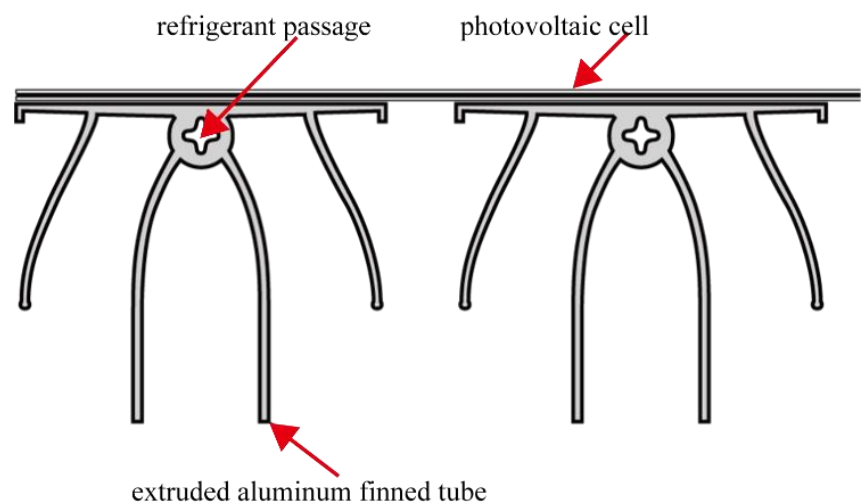

Fig. 4. Cross section of SSHP outdoor panel

Table 1. Specifications of the SSHP module

\begin{tabular}{|c|c|}
\hline Panel Angle & $30^{\circ}$ facing due south \\
\hline Panel Area & About 8 \\
\hline Thermal Output & $5 \mathrm{~kW}$ (nominal) \\
\hline $\begin{array}{c}\text { Electricity Generation } \\
\text { Capacity }\end{array}$ & $1.2 \mathrm{~kW}$ (nominal) \\
\hline Refrigerant & R-32 \\
\hline
\end{tabular}

\subsection{Experiment overview}

The winter experiments were carried out at the Kashiwa Campus of the University of Tokyo throughout the month of March in 2018. A floor-heating heat pump (FHHP) was the only heating equipment in operation. SSHP and GHE were used as heat-source equipment to meet the FHHP heat demands by transferring heat through the thermal network. We adopted two different control schedules for the SSHP heat collection operation, as shown in Figure 5. The first schedule was used from March 1 to 9. The circulation pump was run during the daytime (from 8:00 to 18:00) to heat the circulating water. When the inlet water temperature of the water loop dropped to $15^{\circ} \mathrm{C}$, the SSHP began continually collecting heat until the inlet water temperature of the water loop exceeded $17{ }^{\circ} \mathrm{C}$. During the night-time, i.e., from 18:00 to 8:00 on the next day, the start/stop temperature of the SSHP was lowered, and heat was collected through the GHE to exploit underground heat. In addition, the circulation pump was interlocked with the SSHP compressor. The second schedule was used from March 10 to March 31. We changed the daytime period in the second schedule in order to optimally exploit solar radiation. Furthermore, the stop temperature of the SSHP was reduced to $10{ }^{\circ} \mathrm{C}$. For an in-depth explanation of the control schedule of the system, refer to [4].

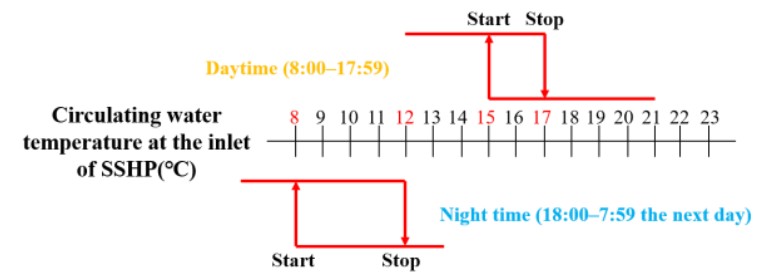

(a) March 1 to 9 control schedule

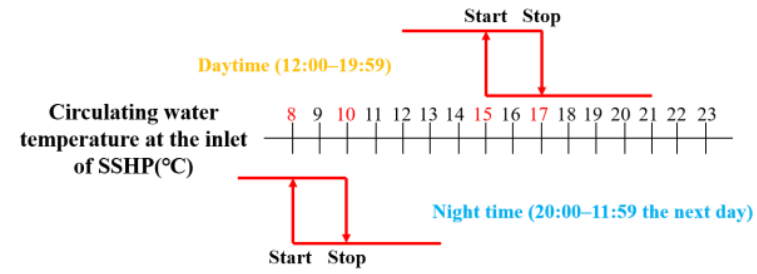

(b) March 10 to 31 control schedule

Fig. 5. Control schedules for SSHP heat collection operation during the month of March

The SSHP circulation pump was set to operate for a duration of 27 minutes and subsequently suspend operation for a period of three minutes in order to sample the circulating water temperature. Since the circulating water is tap water to which antifreeze was not added, this operation strategy is presumed to be able to prevent the circulating water temperature from freezing regardless of the time of day in winter.

Several data loggers were installed in different locations to measure a comprehensive amount of data from the SSHP for experimental analysis and ANN modeling. The following measurements were performed:

a. Water flow rate measured on the water loop side of the plate-type heat exchanger by using a flowmeter (accuracy: $\pm 1.6 \%$ of full scale)

b. Inlet and outlet water temperature of the water loop measured at the inlet and outlet of the plate-type heat exchanger by using platinum resistance bulbs (Pt-100, 
accuracy: $\left.\pm(0.15+0.002|\mathrm{t}|){ }^{\circ} \mathrm{C}\right)$

c. Outdoor temperature and relative humidity measured at a weather station near the RE house by using a thermohygrometer (temperature measurement accuracy: \pm $(0.226-0.0028 \mathrm{t}){ }^{\circ} \mathrm{C}$

d. Solar radiation, long-wave solar radiation from the sky, and long-wave solar radiation from the ground measured at the weather station by using a pyranometer (accuracy: non-linearity $<1 \%$ )

e. Wind speed (accuracy: $0.3 \mathrm{~m} / \mathrm{s}$ when wind speed is less than $35 \mathrm{~m} / \mathrm{s}$ ) and wind direction (accuracy: $3^{\circ}$ when wind speed exceeds $1 \mathrm{~m} / \mathrm{s}$ ) measured at the weather station by using a weather instrument

f. Electricity consumption of the compressor measured by using a power monitor (accuracy: $\pm 2.0 \%$ of full scale \pm 1 digit)

g. Inlet and outlet refrigerant temperature of the SSHP panel measured at the inlet and outlet of the refrigerant passage, and the average was used to represent the evaporation temperature of the SSHP

All the above measurements were performed under the condition of a 5-s sampling interval, and the average value per minute was calculated.

In order to remove the data collected in an unstable state of operation, such as the periods of sudden startup and shutdown of the SSHP compressor, $Q_{\text {sshp }}$ (amount of heat produced by the SSHP condenser), $V_{\mathrm{w}}$ (water flow rate), and $W_{\text {com }}$ (amount of electricity consumed by the SSHP compressor) were used as the criteria to judge whether the raw data should be deleted or retained; Table 2 lists the values for these criteria. Consequently, a data set totaling 4598 data points was obtained. Heat production and the coefficient of performance (COP) of the SSHP were calculated by Equations (1) and (2). The remaining input parameters did not require any calculations subsequent to measurement.

$$
\begin{gathered}
Q_{\text {sshp }}=V_{\mathrm{w}} \times \rho_{\mathrm{w}} \times c_{\mathrm{w}} \times\left(T_{\mathrm{w}, \mathrm{o}}-T_{\mathrm{w}, \mathrm{i}}\right) \\
C O P_{\text {sshp }}=Q_{\text {sshp }} / W_{\text {com }}
\end{gathered}
$$

Table 2. Criteria used to remove unstable data

\begin{tabular}{|c|c|}
\hline Parameter & Value \\
\hline$Q_{\text {sshp }}$ & $\geq 4.5$ \\
\hline$V_{\mathrm{w}}$ & $\geq 25$ \\
\hline$W_{\text {com }}$ & $\geq 0.1$ \\
\hline
\end{tabular}

\section{ANN modeling for performance prediction}

\subsection{ANN description}

An ANN is regarded as a type of black-box model that mimics human brain function to build a mapping relationship between inputs and outputs via interactions between artificial neurons in different layers. ANNs have recently been garnering attention and have been successfully applied to practical problems in various fields owing to the following advantages: 1) their ability to approximate complicated nonlinear relationships and extract knowledge within input and output data, 2) their low computational cost, and 3) their ability to establish relationships between the inputs and outputs with good prediction accuracy. In HVAC research, ANNs are used to model the performance of thermal systems and to predict the energy consumption of a building. The most commonly implemented ANN is the feedforward neural network (FNN). A typical FNN is shown in Figure 6; it consists of three layers: an input layer, a hidden layer, and an output layer. Each layer comprises neurons connected to others by an adaptive weight that is adjusted by training pairs of input and output data. The neurons in the input layer are typically referred to as variables or features, and they provide outside information to the network without any computation. The neurons in a hidden layer are placed between the input and output layers, and thus have no direct connection to outside information. The numbers of hidden layers and hidden neurons represent the ability of the ANN to model intricate nonlinear relationships. However, adding too many hidden layers and hidden neurons is not advisable as it results in overfitting, which means that the ANN overfits accidental regularities in the data, such as data noise, and thus harm the generalization performance of the ANN. The neurons in the output layer represent the target (predicted) values. The error is computed in the output layer and repeatedly distributed back through the network layers to update the weights in order to minimize the error, which is typically referred to as the backward propagation of error. For an in-depth introduction to the theory of ANNs, refer to $[10,16]$.

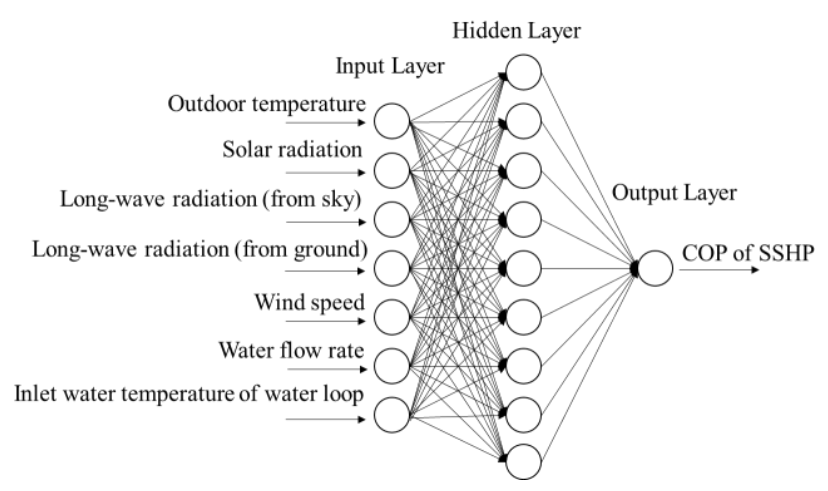

Fig. 6. Architecture of an FNN

\subsection{Definition of the input and output parameters}

As previously mentioned, in this study, the following four output parameters were adopted to describe the thermal performance of SSHP during winter: the outlet water temperature of the water loop, electricity consumption, heat production, and the COP. To boost the prediction accuracy of the ANN, prior to determining the input parameters of the ANN, we analyzed factors that may influence the performance of the SSHP based on the analysis of the measurement results and previous studies. By considering the SSHP as a black box with its own particular characteristics, and which is influenced by the ambient environment, the influencing factors can be generalized as weather conditions, 
water loop characteristics, and the compressor characteristics of the SSHP.

\subsubsection{Weather conditions}

Figure 7 shows the March 2 measurement results for the SSHP. In this figure, (a) and (b) clearly show that, from the start of operation, the COP of the SSHP fluctuated as the solar radiation and outdoor temperature varied. Thus, it can be inferred that the COP of the SSHP was mainly influenced by solar radiation and outdoor temperature. In addition, it was found that long-wave solar radiation influenced SSHP performance, although the level of influence was minimal [11]. According to the study by Kong et al., depending on the relationship between the surface temperature of an SSHP panel and the outdoor temperature, the wind speed may have positive or negative effects on the performance of the SSHP [12]. This phenomenon is illustrated in Figure 7 (d). When the intensity of solar radiation exceeded the maximum thermal output of the SSHP (about $5 \mathrm{~kW}$ here), the surface temperature of the SSHP panel tended to become higher than the outdoor temperature. At this time, the rising wind speed would induce a large amount of heat loss and cause the SSHP collection efficiency to decrease. In contrast, when the intensity of solar radiation was low because of weather conditions such as a cloudy day, the surface temperature of the SSHP panel tended to drop below that of the outdoor temperature. At this time, the rising wind speed would enhance the rate of heat transfer to help the SSHP panel acquire more heat from the ambient air, thereby improving the COP and collection efficiency of the SSHP. There are few studies on how much wind direction and relative humidity affect the thermal performance of an SSHP. Thus, in this research, to examine the respective degrees of the influence, both wind direction and relative humidity were incorporated into the ANN as input parameters.

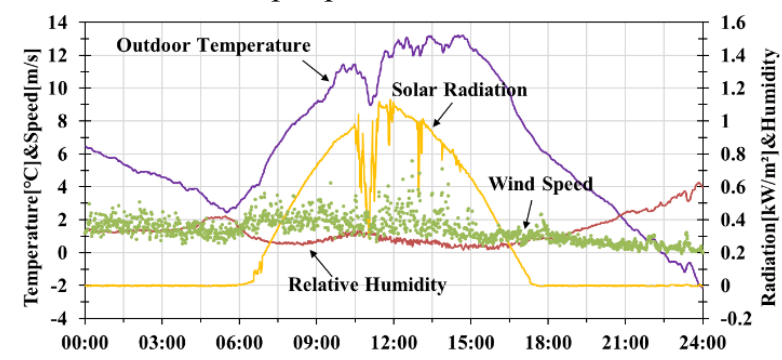

(a) Weather conditions on March 2

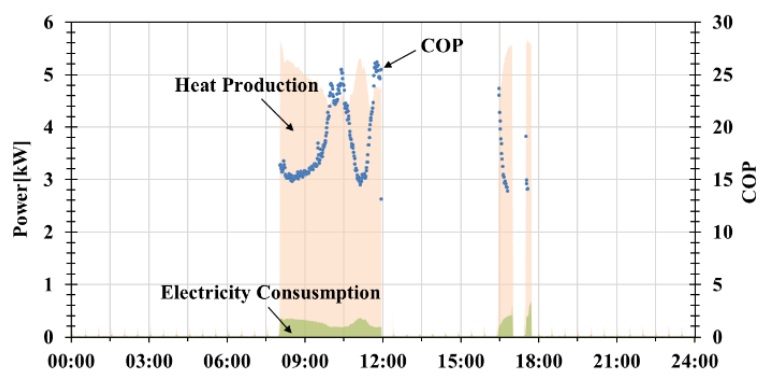

(b) SSHP performance fluctuation

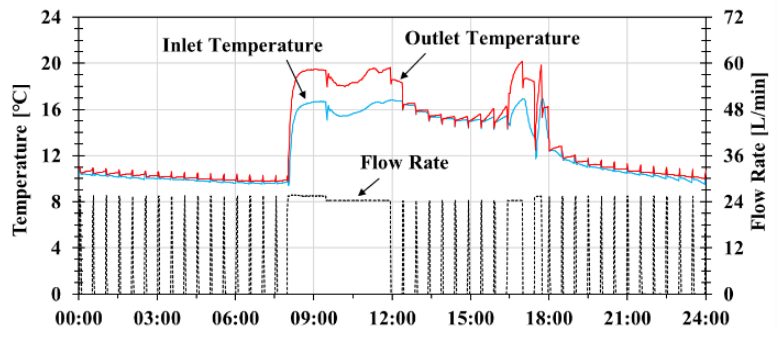

(c) Changes in the SSHP water loop

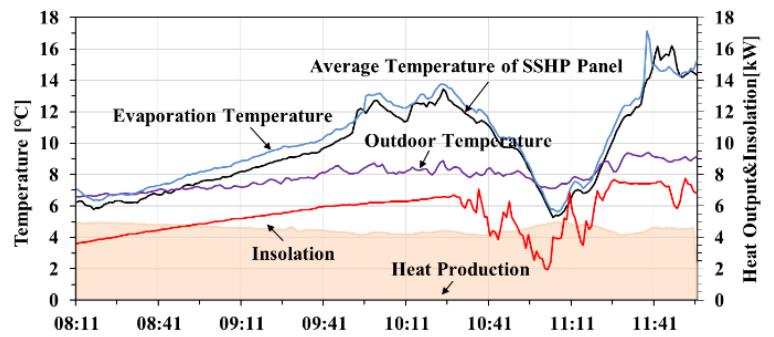

(d) Changes in the evaporation and surface temperatures of an insulated SSHP panel

Fig. 7. Measurement results for March 2

\subsubsection{Water loop characteristics}

Since the inlet water temperature and water flow rate of the water loop tended to significantly affect the condensation temperature of the SSHP compressor, these factors were implemented as two of the input parameters.

\subsubsection{SSHP compressor characteristics}

The speed of the compressor is directly related to the amount of circulated refrigerant, which determines how much heat the SSHP can output. In addition, in our experiments, the speed of the compressor was automatically adjusted according to the changes in the average evaporation temperature of the SSHP panel; thus, the average evaporation temperature was used as one of the ANN input parameters.

Figure 8 shows the initial input and output parameters that were introduced to the ANN in order to develop a black-box model based on the above analysis.

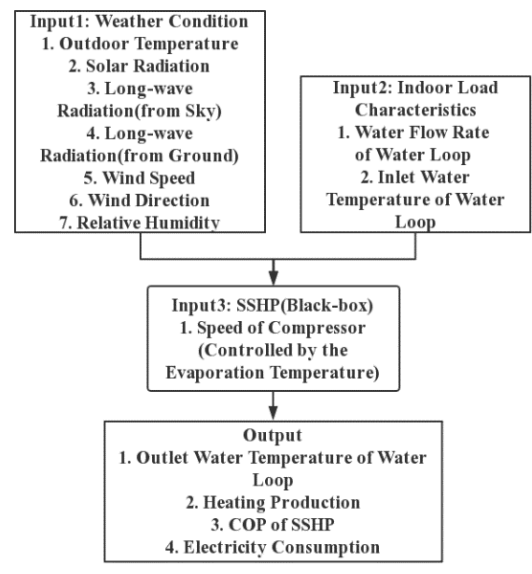

Fig. 8. Black-box model of the SSHP 


\subsection{Development of the ANN}

In this study, we used the neural network toolbox of MATLAB2017a to construct the ANN for the SSHP winter thermal performance model. A three-layer FNN (input layer - hidden layer - output layer) was implemented as the initial ANN configuration because, according to the universal approximation theorem, an FNN with a single hidden layer with sufficient neurons can approximate any complicated function that requires continuous mapping between finite spaces [13]. The number of neurons in the input layer was set to equal the number of the input parameters introduced to the ANN; this number was varied, and it is discussed in Section 4. The number of neurons in the hidden layer was set to an initial value of 10 . The number of neurons in the output layer was set to 1, as this means that an ANN was created and trained for every output parameter. The LevenbergMarquardt algorithm was employed as the optimization method, as it is highly recommended as a tool to train an ANN constructed by using the neural network toolbox owing to its high run speed and prediction accuracy [14]. The available data collected in the winter field experiment (4598 data) was split into the following three data subsets: the training data set ( $70 \%, 3219$ data points), the validation data set (15\%, 689 data points), and the test data set $(15 \%, 690$ data points). The training data set was used to optimize the weights of the ANN in order to minimize the error between the predicted output and target value. The validation data set was used to discontinue network training to prevent overfitting if the network performance on the validation data set failed to improve, or remained the same for six epochs in a row; this method is referred to as early-stopping [14]. The validation data set was also used to optimize input parameter selection in order to maximize ANN accuracy, while also enabling investigation into the effects of the input parameters on SSHP performance. The remaining test data set was used to assess the generalization performance of the best ANN based on the following three metrics: the coefficient of determination $\left(\mathrm{R}^{2}\right)$, root mean squared error (RMSE), and mean absolute error (MAE). Before training the ANN, we implemented random initialization to randomize the weight values within the interval of $[-1,1]$. The activation functions implemented in the hidden and output layers were a hyperbolic tangent sigmoid function and linear function, respectively. In addition, data normalization was also implemented during training because the ranges of the values of the input data points were significantly different.

\section{Results and discussion}

\subsection{Selection of the best combination of input parameters}

To realize a highly accurate ANN model and simultaneously investigate the effects of the input parameters on the performance of the SSHP, six case studies with different combinations of input parameters were carried out by using the validation data set; the case studies are outlined in Table 3.
Table 3. Case studies

\begin{tabular}{|c|c|}
\hline Case & Input parameters \\
\hline Case 1 & $T_{\mathrm{a}}, I_{\mathrm{s}}, \nu_{\mathrm{w}}, J_{\mathrm{a}}, J_{\mathrm{t}}, T_{\mathrm{w}, \mathrm{i}}, V_{\mathrm{w}}$ \\
\hline Case 2 & Case $1, \beta_{\mathrm{w}}$ \\
\hline Case 3 & Case $1, \varphi_{\mathrm{a}}$ \\
\hline Case 4 & Case $1, \beta_{\mathrm{w}}, \varphi_{\mathrm{a}}$ \\
\hline Case 5 & Case $1, T_{\mathrm{r}}$ \\
\hline Case 6 & Case $1, \beta_{\mathrm{w}}, \varphi_{\mathrm{a}}, T_{\mathrm{r}}$ \\
\hline
\end{tabular}

The results of all of the case studies are shown in Figure 9. Focusing on the results from Case 1 - Case4, it was found that $\beta_{\mathrm{w}}$ (wind direction) and $\varphi_{\mathrm{a}}$ (relative humidity) only minimally influenced the accuracies of all output parameters. Conversely, comparing the results from Case 5 and Case6, i.e., the case studies in which the average evaporation temperature of the SSHP panel $T_{\mathrm{r}}$ was taken into account, to the results from Case $1 \sim$ Case 4 revealed that the prediction accuracy of the ANN model could be considerably increased by introducing $T_{\mathrm{r}}$, which was used to automatically control the speed of the SSHP compressor in our experiment. We subsequently selected the best ANNs based on these results and assessed their generalization performance in the test data set. The test data results yielded the following results for water-loop outlet water temperature, electricity consumption, heat production, and COP, respectively: RMSE values of $0.045^{\circ} \mathrm{C}, 0.027 \mathrm{~kW}, 0.082 \mathrm{~kW}$, and $1.358 ; \mathrm{R}^{2}$ values of 0.999 , $0.974,0.953$, and 0.968 ; MAE values of $0.028^{\circ} \mathrm{C}, 0.017 \mathrm{~kW}$, $0.050 \mathrm{~kW}$, and 0.877 . These reported results indicate that the ANN model developed in this study can achieve relatively high SSHP winter performance prediction accuracy.

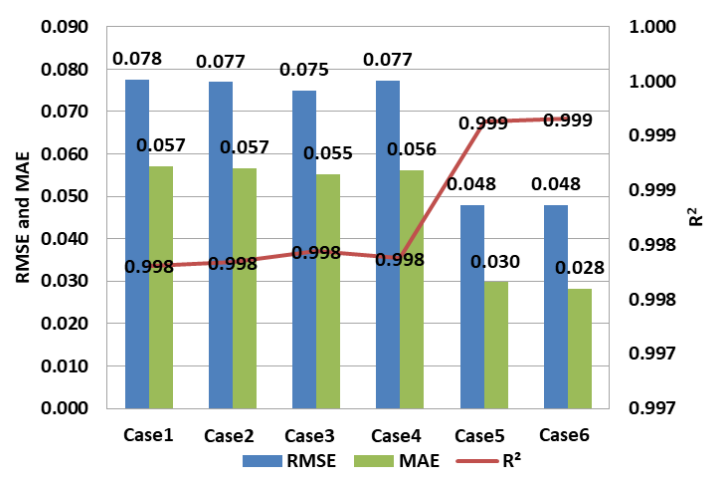

(a) Outlet water temperature of the water loop

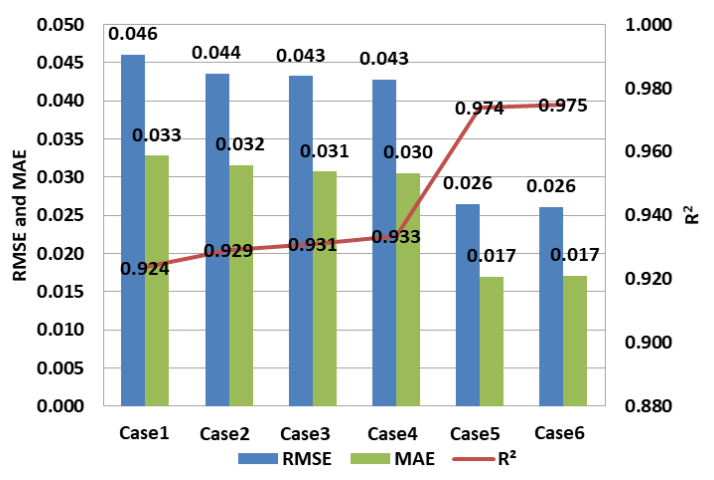

(b) Electricity consumption 


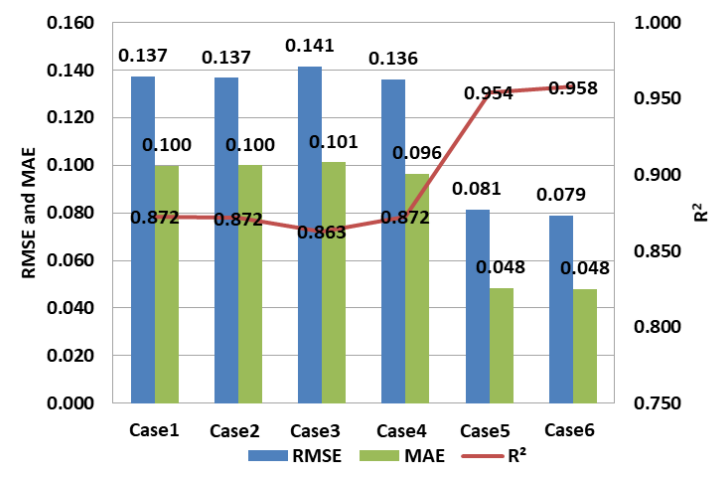

(c) Heat production

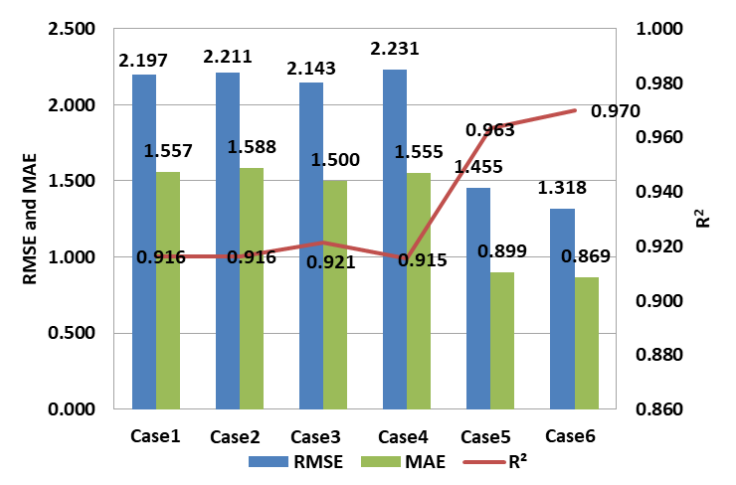

(d) SSHP COP

Fig. 9. Comparison of the 10-epoch-averaged values for the three metrics calculated as based on the validation data set for all six SSHP thermal performance case studies

\subsection{Learning curves: how the training data size affects the generalization performance of the ANN}

Generally, the generalization performance of models can be improved by training models on a large data set because a large data set reduces overfitting and thus increases the extrapolation accuracy beyond the training data. In this study, we used learning curves to clarify the relationship between the training data size and the generalization performance of the ANN; the results are shown in Figure 10. The training data size and generalization performance (e.g., RMSE, or $\mathrm{R}^{2}$ metric used to represent the generalization performance of models), or training error, were plotted as learning curves [15-16]. Initially, the entire training data set $(70 \%, 3219$ data points) was split into 16 subsets $(\mathrm{N}=20,30,50,100,200$, $300,500,700,1000,1200,1500,1800,2000,2500,3000$, and $3219)$ that were used to respectively train 16 ANNs. Then, the validation data set $(15 \%, 689$ data points) was used to tune the parameters of the artificial neurons. Lastly, each ANN was evaluated by using the remaining test data set $(15 \%, 690$ data pints). Since each training epoch produces a different ANN even when the same training data is used, to minimize statistical variation, the calculated results were the respective averages of 100 epochs. From the learning curves shown in Figure 10, it can be seen that, with the exception of one outlier in the third subset (50 data points) that shows an opposite trend caused by statistical fluctuations, the test error curve initially rapidly decreases in response to an increased training data size. Conversely, the training error curve shows an initial rapid increase. This makes sense, because it is harder to fit a larger data set for a fixed-capacity ANN. The figure also shows that the effects of incrementally increasing the training data size seems to gradually diminish until both curves begin to plateau. More specifically, convergence can be observed when the data size is approximately 2000-2500, with the slopes of both curves approaching zero; this means that a data size above 3219 would not substantially improve the generalization performance of the ANN. Moreover, with the largest data size, the gap between the training error and test error is small, indicating that the proposed ANN has low variance.

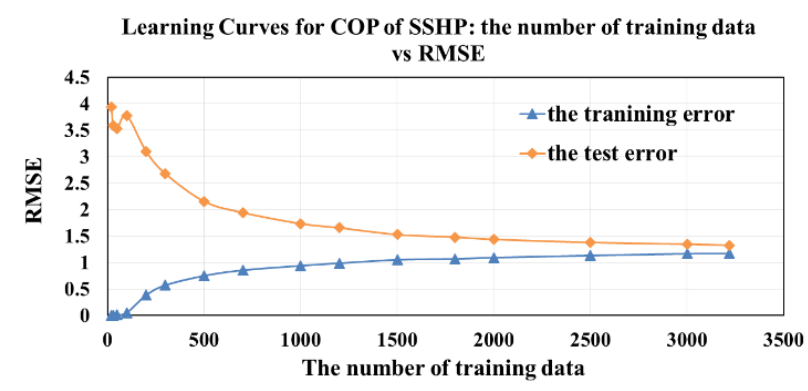

Fig. 10. Learning curves for the SSHP COP showing how the training data size affects the generalization performance of ANN

\section{Summary}

In this study, an ANN was developed and used to model the winter thermal performance of a novel direct-expansion solar-assisted heat pump. We considered the SSHP to be a black box that is influenced by the ambient environment and its own characteristics in order to determine the input and output parameters of the ANN model. Consequently, the following three input parameters were considered: weather conditions, water loop characteristics, and the SSHP compressor characteristics. Based on the results of previous studies and our experimental analysis, we analyzed how each input parameter would influence the winter thermal performance of the SSHP. The following four output parameters were adopted to evaluate the performance: the water-loop outlet water temperature, electricity consumption, heat production, and the COP. The data collected during the winter field experiment was partitioned into the following three data sets: 1) the training data set used to optimize the ANN weights in order to minimize the error between the predicted output and target value, 2) the validation data set used to tune the parameters of the ANN to prevent overfitting or facilitate model selection, and 3) the test data set used to assess the generalization performance of the fully trained ANN. To maximize accuracy of the ANN model and investigate the effects of the input parameters on the performance of the SSHP, case studies with different combinations of input parameters were carried out and evaluated in terms of the following metrics: $\mathrm{R}^{2}, \mathrm{RMSE}$, and MAE, which were calculated as based on the validation data set. The results from the case studies showed that the prediction accuracy of the ANN model could be considerably 
improved by taking into account the average refrigerant temperature of the SSHP panel. However, it was apparent that the wind direction and relative humidity had minimal influence on the accuracies of all output parameters. Additionally, learning curves were introduced in this study to clarify the relationship between the training data size and the generalization performance of the $\mathrm{ANN}$, as these results could help us determine whether it is necessary to spend extra money and time to acquire additional data in order to further improve the generalization performance of the ANN. Finally, the best ANNs were selected and evaluated as based on the test data set by using the above-mentioned metrics. The predicted performance results indicated that the proposed ANN model is able to predict the winter thermal performance of an SSHP with relatively high accuracy. Because the ANN developed in this study can establish relationships between the inputs and outputs with better prediction accuracy than a physics-based model, this research is expected to help researchers easily predict the thermal performance of a directexpansion solar-assisted heat pump such as the SSHP under different conditions. Moreover, this type of ANN-based model can be effectively integrated into control techniques, such as the model predictive control technique, because it has low computational cost and its weights can be adjusted to suit the dynamic characteristics of HVAC equipment based on the collected data [17].

Nomenclature

$T_{\mathrm{a}}$ : Outdoor temperature $\left[{ }^{\circ} \mathrm{C}\right]$

$I_{\mathrm{s}}$ : Solar radiation $\left[\mathrm{W} / \mathrm{m}^{2}\right]$

$v_{\mathrm{w}}$ : Wind speed $[\mathrm{m} / \mathrm{s}]$

$J_{\mathrm{a}}$ : Long-wave solar radiation (from sky) $\left[\mathrm{W} / \mathrm{m}^{2}\right]$

$J_{\mathrm{t}}$ : Long-wave solar radiation (from ground) $\left[\mathrm{W} / \mathrm{m}^{2}\right]$

$\beta_{\mathrm{w}}$ : Wind direction $\left[{ }^{\circ}\right]$

$\varphi_{\mathrm{a}}$ : Relative humidity [\%]

$T_{\mathrm{r}}$ : Average refrigerant temperature of SSHP panel $\left[{ }^{\circ} \mathrm{C}\right]$

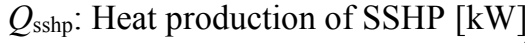

$V_{\mathrm{w}}$ : Water flow rate of water loop $\left[\mathrm{m}^{3} / \mathrm{s}\right]$

$\rho_{\mathrm{w}}$ : Density of the circulating water $\left[\mathrm{kg} / \mathrm{m}^{3}\right]$

$c_{\mathrm{w}}$ : Specific heat of the circulating water $[\mathrm{J} /(\mathrm{kg} \mathrm{K})]$

$T_{\mathrm{w}, \mathrm{o}}$ : Outlet water temperature of water loop $\left[{ }^{\circ} \mathrm{C}\right]$

$T_{\mathrm{w}, \mathrm{i}}$ : Inlet water temperature of water loop $\left[{ }^{\circ} \mathrm{C}\right]$

$W_{\text {com: }}$ : Electricity consumption of the compressor of the SSHP $[\mathrm{kW}]$

Acknowledgements: This development is a joint project with the National Energy and Industrial Technology Development Organization (NEDO) "Renewable Energy Thermal Utilization Technology Development". We extend our appreciation to everyone concerned.

\section{References}

1. P.A. Owusu, S. Asumadu-sarkodie, Cogent Eng. 15, 1 (2016).

2. T. Hino, R. Ooka, Proc. Clima2013 (REHVA, Prague, Czech Republic, 2013).

3. X.Q. Zhai, M. Qu, X. Yu, Y. Yang, and R.Z. Wang,

Renew. Sustain. Energy Rev. 15, 3133 (2011).

4. L. Dai, S. Li, L. DuanMu, X. Li, Y. Shang, M. Dong, Appl. Therm. Eng. 75, 325 (2015).
5. M.Z. Liu, K. Wen, Proc. Clima2019 (REHVA, Bucharest, Romania, 2019). (submitted)

6. T. Hino, R. Ooka, Proc. EnerSTOCK2018 (Adana, Turkey, 2018).

7. Z. Afroz, G.M. Shafiullah, T. Urmee, G. Higgins, Renew. Sustain. Energy Rev. 83, 64 (2018).

8. M. Mohanraj, S. Jayaraj, C. Muraleedharan, Appl. Energy 86, 1442 (2009).

9. N. Gunasekar, M. Mohanraj, V. Velmurugan, Energy 93, 908 (2015)

10. M. Mohanraj, S. Jayaraj, C. Muraleedharan, Renew.

Sustain. Energy Rev. 16, 1340 (2011).

11. T. Hino, R. Ooka, S. Yoshida, K. Kodama, IEA Heat

Pump Conference (Montreal, Canada, 2014)

12. X.Q. Kong, D. Zhang, Y. Li, Q.M. Yang, Energy 36, 6830 (2011).

13. B.C. Csáji, Approximation with Artificial Neural Networks (Faculty of Sciences, Etvs Lornd University, Hungary, 2001).

14. M.H. Beale, M.T. Hagan, H.B. Demuth, Deep Learning Toolbox User's Guide (The MathWorks Inc., Natick, MA 2018)

15. C. Cortes, L.D. Jackel, S.A. Solla, V. Vapnik, J.S.

Denker, Adv. Neural Inf. Process. Syst. 6, 327 (1994).

16. I. Goodfellow, Y. Bengio, A. Courville, Deep Learning (MIT Press, 2016)

17. A. Afram, F. Janabi-Sharifi, A.S. Fung, K. Raahemifar, Energy Build. 141, 96 (2017) 